\title{
Infrared Tympanic Thermometry
}

Gauri Mankekar, Chief Medical Officer, A. J.B.M. ENT Hospital, Mumbai

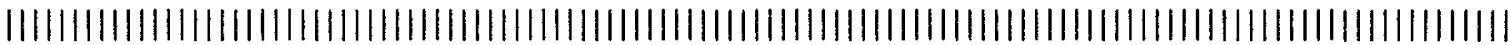

C onventionally body temperature has been measured using glass mercury and electronic thermometers. Such devices measure the peripheral body temperature in the sublingual pockets, in the rectum or under the arms. peripheral temperatures, so measured, can be influenced by the local conditions such as :-

1. Oral temperatures are influenced by thermometer placement, recent ingestion of hot or cold liquids and rapid breathing;

2. rectal temperatures may vary because of poor blood supply to this area, the insulating effects of stool and the presence of heat producing organisms.

3. axillary temperatures can be influenced by vasodilatation and constriction caused by the body's response to temperature changes.

4. incorrect thermometer placement.

Thanks to technological advantages, it is pos- sible today, to measure body temperature at the tympanic membrane using infrared technology. A probe inserted into the ear canal measures the infra-red energy that naturally radiates from the tympanic membrane and surrounding tissues. A microprocessor connected to the ear probe analyses, converts and displays the temperature within one second.

The accuracy of this method is attributed to the tympanic membrane's close anatomic proximity to the hypothalamic thermore-gulatory centre and common blood supply from the carotid artery. Since temperature is displayed within one second, the ear thermometer (which is marketed in the shape of an otoscope) offers a les stressful and faster method of temperature measurement, Therefore, it is especially useful in children. Neither cerumen nor otitis media significantly affect the readings. So, infrared tympanic thermometry offers a relatively noninvasive, comfortable and faster option compared to conventional thermometry.

\section{|||||||||||||||||||||||||||||||||||||||||||||||||||||||||||||||||||||||||||||||||||||||||||||||||||||||||||||| $\mid$}

\section{References}

1. M. Benzinger (1969.) Tympanic thermometry in Surgery and anaesthesia. JAMA : August 25.

2. B. Kelly \& D. Alexander (1991.) Effect of Otitis media on infrared tympanic thermometry, Clinical Paediatrics, April.

3. S. Pransky: April (1991.) The impact of technique and conditions of the tympanic membrane upon intrared tympanic termometry, Clinical Paediatrics. 